\title{
Adultos mayores en riesgo de Covid-19 y sus vulnerabilidades socioeconómicas y familiares: un análisis con el ENASEM
}

\section{Older adults at risk of Covid-19 and their socioeconomic ans family vulnerabilities: an analysis with MHAS}

\author{
César González-González*, Karina Orozco-Rocha*, \\ Rafael Samper-Ternent** y Rebeca Wong** \\ Universidad de Colima, México*, \\ The University of Texas Medical Branch, Estados Unidos**
}

\begin{abstract}
Resumen
El objetivo de este manuscrito es analizar las características socioeconómicas y familiares de personas de 50 años o más que están en riesgo alto de dar positivo a Covid-19, ser hospitalizado o intubado en México. Para ello se utilizan dos fuentes de información, la base de datos de la Secretaría de Salud sobre Covid-19 y el Estudio Nacional de Salud y Envejecimiento en México, en la primera se estiman modelos de regresión para calcular el riesgo y se aplican en la segunda para analizar las vulnerabilidades socioeconómicas y familiares. Los resultados señalan que el sexo y la edad son fuertes predictores de riesgo alto. Los de menos escolaridad, los que trabajan sin disponer un local, los que perciben su situación económica como regular o mala, así como los que viven en hogares unipersonales, reciben ayuda económica y no económica de sus hijos se presentan como un grupo altamente vulnerable a Covid-19 y sus repercusiones.
\end{abstract}

Palabras clave: Covid-19, envejecimiento, ENASEM, vulnerabilidad, familia.

Abstract

The objective of this manuscript is to analyze the socioeconomic and family characteristics of population aged 50 years and older who are at high risk of testing positive for Covid-19, being hospitalized, or intubated in Mexico. We used two sources of information, the database of the Ministry of Health on Covid-19 and the Mexican Health and Aging Study in Mexico. We use the first to estimated regression models to calculate the risk of Covid-19, and these are applied in the second to analyze socioeconomic and family vulnerabilities. Results show that gender and age are strong predictors of high risk. Those with less schooling, those who work without a fixed physical place, those who perceive their economic situation as fair or bad, those who live in single-person households, and those who receive financial and no-financial help from their children are presented as a highly vulnerable group to Covid-19 and its repercussions.

Keywords: Covid-19, Aging, MHAS, vulnerability, family. 


\section{INTRODUCCIÓN}

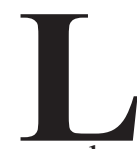

a pandemia de Covid-19 ha impactado la salud de la población mundial; en particular a las personas de 60 años o más, o aquellos que tienen condiciones de salud como enfermedad cardiaca o pulmonar, diabetes o condiciones que afectan su sistema inmune. Las Personas Adultas Mayores (PAM) en México son especialmente sensibles a Covid-19, pues una parte importante de este grupo poblacional de edad 60 o más presenta las condiciones que la hacen vulnerable a esta enfermedad; 24 por ciento presenta diabetes, poco menos de la mitad hipertensión, 5.1 por ciento ataque al corazón y 4.7 por ciento enfermedad pulmonar. Además, los adultos mayores de las cohortes más viejas pueden presentar síntomas atípicos y anomalías en múltiples órganos comparado con los adultos mayores más jóvenes, lo que complica aún más su estado de salud (Guo et al., 2020).

A nivel mundial la tasa de positividad para Covid-19 presenta amplias diferencias entre países y regiones, pues depende del sistema de vigilancia epidemiológica que se implementa en cada país y del número de pruebas que se realizan. En México, la tasa de positividad reportada hasta ahora es 45.8 por ciento, ${ }^{1}$ una de las más altas a nivel mundial; mientras que en países como Australia, Corea del Sur y Uruguay tienen una tasa de positividad menor a uno por ciento (Ritchie et al., 2020). En México se realizan 83 pruebas por cada millón de habitantes, y un dato que resulta interesante es que se practican 2.2 pruebas por cada caso confirmado, ${ }^{2}$ y las pruebas se realizan sólo en aquellos que presentan síntomas graves asociados a Covid-19.

No solamente preocupa la positividad del nuevo virus, también la situación grave de salud y su letalidad en particular en las personas adultas mayores. Según datos publicados por Mallapaty (2020) a nivel mundial, por cada mil personas infectadas con el coronavirus y que tienen menos de 50 años, la probabilidad de fallecer son casi nulas; entre los 50 y 69 años, morirán alrededor de cinco por cada mil; y para los que tienen 70 años o más, se estima que morirán 116 personas por cada mil. Los estudios revelan que la edad es el predictor más fuerte del riesgo de muerte de una persona infectada por Covid-19. Justo en ese sentido, según los datos de la Secretaría de Salud, la población de 50 años o más representa 37.3 por ciento del total de casos confirmados y 81.9 por ciento de las muertes ocu-

\footnotetext{
Datos al 1 de septiembre de 2020.

2 Datos al 1 de septiembre de 2020.
} 
Adultos mayores en riesgo de Covid-19 y sus vulnerabilidades socieconómicas y familiares ... / C. GONZÁLEZ GONZÁLEZ et al.

rridas por Covid-19 en México (Gobierno de México, 2020). ${ }^{3}$

Pero la afectación del estado de salud no ha sido el único impacto de la pandemia del Covid-19, también ha tenido implicaciones desfavorables en al ámbito socioeconómico y existe incertidumbre sobre su evolución en el futuro cercano. A partir de la pandemia, las economías y sociedades se han paralizado, un evento comparable a una situación de guerra (CEPAL, 2020a). En este panorama, las personas adultas mayores son consideradas como uno de los grupos vulnerables a los impactos de la recesión económica, con efectos directos sobre su calidad de vida y el bienestar (Fenge et al., 2012). Más aún, la pandemia del Covid-19 llega a Latinoamérica y a México en un momento de bajo crecimiento económico, alta desigualdad y vulnerabilidad económica, y se prevé que la región tenga una histórica contracción económica no vista desde hace 120 años (CEPAL, 2020c). Ante un impacto de la crisis económica, identificar las vulnerabilidades socioeconómicas en los adultos mayores a través de indicadores empíricos es importante para una adecuada asignación de recursos y programas de atención hacia quienes tienen mayores riesgos de enfrentar una situación adversa (Fernandes et al., 2017) como la enfermedad del Covid-19, y contar con recursos limitados para sobrellevar la crisis económica actual.

Lo complicado de la pandemia, las características de riesgo en salud y de vulnerabilidad socioeconómica que presentan las personas adultas mayores, así como las relaciones familiares presentes en la sociedad mexicana nos hacen preguntarnos ¿Con qué recursos familiares y condiciones socioeconómicas cuentan las Personas Adultas Mayores en México para hacer frente a una enfermedad emergente como el Covid-19 y la subsecuente crisis económica?

Este trabajo tiene tres objetivos i) identificar las variables asociadas a dar positivo a Covid-19, ser hospitalizado o ser intubado en los individuos que acudieron a hacerse la prueba de Covid-19; ii) construir un índice de riesgo de positividad con los resultados obtenidos, y iii) examinar cómo los niveles más altos de riesgo por Covid-19 están asociados a las vulnerabilidades socioeconómicas de los adultos mayores y los recursos familiares con los que dispone. El objetivo global es explorar las características de aquellos que están en mayor riesgo de Covid-19, es decir, identificar dentro de los vulnerables a los más vulnerables por no contar con las condiciones socioeconómicas o familiares necesarias para enfrentar la enfermedad y la crisis económica. Pues en un país con 23 millones de personas de 50 años o más, que representa 19.6 por ciento de la población total (INEGI, 2015),

\footnotetext{
${ }^{3}$ https://coronavirus.gob.mx/datos/\#DOView
} 
es importante generar conocimiento que permita minimizar los riesgos. Estudios como el que se está proponiendo son de suma importancia para analizar los recursos económicos y familiares con que cuentan las personas adultas mayores para hacer frente a la pandemia del Covid-19, en particular su asociación con el riesgo de resultar positivo a dicha enfermedad, de tener una grave situación de salud, y la manera en que puede hacer frente a los impactos negativos de la pandemia.

Una vez establecida la pertinencia del estudio, el manuscrito está integrado por cinco secciones. En la primera se presenta el panorama del Covid-19, los riesgos de contagio y complicaciones del estado de salud de los adultos mayores documentados a nivel internacional; posteriormente, se contextualiza sobre las vulnerabilidades socioeconómicas y familiares en los adultos mayores en México. A partir de dicha revisión se presenta la metodología aplicada la cual hace uso de dos fuentes de información, para examinar el aspecto de riesgo en la enfermedad del Covid-19 y las vulnerabilidades socioeconómicas y familiares. En la cuarta sección se presentan los resultados de los factores de riesgos asociados a dar positivo de Covid-19 y a tener complicaciones; así como las características socioeconómicas y familiares del grupo de adultos mayores con niveles más altos de riesgo de dicha enfermedad. Finalmente, se presenta la discusión y conclusiones.

\section{RiESGo DE CONTAGIO Y COMPLICACIONES DEL ESTADO DE SALUD DE loS ADUltos Mayores aNTe El Covid-19}

En epidemiología se utiliza el enfoque de riesgos para examinar la situación de salud de las personas. Aunque el Covid-19 afecta a toda la población, por las condiciones asociadas con las personas adultas mayores, éstas han sido consideradas como un grupo de alto riesgo (Huenchuan, 2020a). De ahí que en este estudio se analiza el riesgo de las personas adultas mayores de ser contagiadas del Covid-19 y de desarrollar un estado de salud grave que los lleve a ser hospitalizados o incluso intubados.

\section{Riesgo de contagio}

Respecto al riesgo de contagio, se ha documento más en cuanto a la interacción social. La estructura de los hogares es un factor que se ha explorado para analizar el riesgo de infección del Covid-19; aún existe el debate sobre este tema, pues se sospecha que los adultos mayores que residen con sus hijos (sean hogares nucleares o ampliados) tienen mayor riesgo de ad- 
quirir el virus, por la movilidad que presentan los miembros más jóvenes y que representan una fuente de transmisión dentro del hogar (Esteve et al., 2020). Esto es relevante pues 47.9 por ciento de las PAM en México viven en hogares nucleares y 39.8 por ciento en hogares ampliados. Las residencias geriátricas o asilos son otro factor de contagio que ha sido documentado, pues los adultos mayores que viven en asilos presentan mayor riesgo de contagiarse de Covid-19 (Aquino-Camchari, Quispe-Arrieta and Hueman Costillo, 2020); esto se debe a la colectividad en espacios reducidos.

La propia interacción social de las personas adultas mayores también representa un riesgo de contagio; los que tienen enfermedades crónicas que requieren seguimiento continuo, que acuden a hospitales o centros de salud a recibir atención médica de rutina, están más expuestos a un riesgo de contagio si no se les proporciona la asistencia médica adecuada durante una pandemia (Bonanad et al., 2020); garantizar el adecuado aislamiento a través de asistencia remota o telemedicina en países como México resulta complicado por las dificultades en la comunicación telefónica o de video llamadas.

\section{Riesgos de agravar el estado de salud}

Se ha documentado que el riesgo de enfermar de manera grave del Covid-19 se incrementa con la edad, a partir de los 40 años, y en mayor medida a los 60 años o más (Aquino-Camchari, Quispe-Arrieta and Hueman Costillo, 2020; Bonanad et al., 2020; Huenchuan, 2020a). Por sí misma, la morbilidad en los adultos mayores es un elemento de riesgo que compromete y complica su estado de salud (Araujo González, 2015). En la actualidad diversos estudios dan cuenta de manera individual cómo las enfermedades crónicas, incluyendo diabetes, hipertensión, enfermedades cardiovasculares y enfermedades crónicas obstructivas, son consideradas factores de riesgo para enfermar severamente, tener un mal diagnóstico y morir por Covid-19 (Avanoğlu Güler and Akif Öztürk, 2020). La diabetes mellitus se considera un factor de riesgo que agrava y que propicia un mal pronóstico de la enfermedad de Covid-19, por los altos niveles de marcadores inflamatorios, similar a como se ha demostrado previamente con las infecciones epidémicas por betacoronavirus (Avanoğlu Güler and Akif Öztürk, 2020). Las enfermedades cardiovasculares, que tienen una mayor prevalencia entre la población de personas adultas mayores, son un factor de riesgo sustancial que agrava la enfermedad. Los pacientes con cáncer también son susceptibles de tener complicaciones ante el Covid-19, pues por los tratamientos recibidos contra el cáncer su sistema inmunológico se 
encuentra comprometido (Aquino-Camchari, Quispe-Arrieta and Hueman Costillo, 2020).

Los factores fisiológicos de la edad avanzada contribuyen a que se presenten comorbilidades, este es otro factor que agrava la enfermedad del Covid-19 (Aquino-Camchari, Quispe-Arrieta and Hueman Costillo, 2020). Se ha documentado que con la presencia de comorbilidades en los adultos mayores, éstos pueden desarrollar síntomas atípicos y anomalías en múltiples órganos, particularmente las cohortes más ancianas (Guo et al., 2020).

Además, podemos mencionar la obesidad y sobrepeso como factores de riesgo que agravan la enfermedad del Covid-19 (Aquino-Camchari, Quispe-Arrieta and Hueman Costillo, 2020); lo cual representa un dato preocupante pues en México 68 por ciento de los adultos mayores de 50 años o más tiene sobrepeso u obesidad (Rodriguez and Wong, 2019). El género también es un factor de riesgo importante, de agravarse o de morir derivado de la enfermedad de Covid-19. Los hombres tienen casi el doble de probabilidad de morir a causa del coronavirus que las mujeres. Finalmente, las estimaciones de mortalidad para los grupos de mayor edad sugieren que el riesgo de morir por coronavirus también está relacionado con las condiciones de salud subyacentes, la capacidad de los sistemas de atención médica y si el virus se ha propagado entre las personas que viven en centros de atención para ancianos (Mallapaty, 2020).

\section{VULNERABILIDADES SOCIOECONÓMICAS Y FAMILIARES}

Las vulnerabilidades que enfrenta una persona se refieren a los diferentes riesgos a los que está expuesta, y depende del entorno personal, familiar, socioeconómico e incluso político que llegan a debilitar su capacidad para prevenir, hacer frente, resistir y sobreponerse de las situaciones que impactan su bienestar (Araujo González, 2015; Aquino-Camchari, Quispe-Arrieta and Hueman Costillo, 2020).

La vulnerabilidad socioeconómica se asocia a diversas situaciones y dimensiones, principalmente a una mayor edad, menor logro educativo, ser mujer (Andrew et al., 2012), a la carencia e incapacidad de respuesta al riesgo existente asociado con las carencias de recursos materiales, y el acceso a diferentes bienes y servicios (Araujo González, 2015).

En los adultos mayores las vulnerabilidades socioeconómicas se manifiestan en la tendencia decreciente en sus ingresos, en su vinculación a empleos precarios, que los hacen especialmente vulnerables a las fluctuaciones económicas, con impactos directos en su bienestar y calidad de vida (Fenge et al., 2012). Por ejemplo, los empleos que tiene la población de 
65 años o más se caracterizan por ofrecer sueldos bajos y frecuentemente no otorgan las prestaciones necesarias para que la población cuente con acceso a la seguridad social, además los que trabajan lo hace por necesidad $\mathrm{y}$, aun así, sufren carencias y su ingreso es insuficiente para satisfacer sus necesidades básicas (CONEVAL, 2020).

Pero no solamente es el bienestar económico individual, también la vulnerabilidad se ve reflejada en el debilitamiento económico de sus redes familiares o comunitarias, y la exposición a la inseguridad alimentaria, por mencionar algunas dimensiones en el plano personal y familiar. En un momento como el que actualmente se vive en México de recesión económica, con un pronóstico de crecimiento de -6.5 por ciento del PIB en 2020 (CEPAL, 2020b) y con las complicaciones de la pandemia, analizar la vulnerabilidad socioeconómica de los adultos mayores adquiere relevancia. Pues, según estimaciones de la pérdida de empleos, reducción de salarios o pérdida de ingresos es un escenario plausible en un país que no cuenta con seguro por desempleo. Además, en el escenario más conservador se prevé un aumento de la población en situación de pobreza de 41.9 a 46.8 por ciento (CEPAL, 2020c).

Por otra parte, el vínculo entre la vulnerabilidad socioeconómica y el bienestar físico es menos claro y es probable que exista una interrelación compleja entre la economía y la salud. Algunas personas adultas mayores pueden volverse cada vez más vulnerables debido a su creciente mala salud, y las preocupaciones económicas pueden exacerbar esta situación (Fenge et al., 2012). Si el adulto mayor económicamente activo enferma de Covid-19, puede estar seriamente vulnerable en términos económicos, ya que debe dejar de trabajar debido a un comprometido estado salud, y si en su trabajo no dispone de seguridad social que le permita estar en recuperación y recibiendo un salario, su situación económica y de salud puede deteriorarse rápidamente.

Justo con este tema se vincula la vulnerabilidad socioeconómica desde el ámbito institucional, que está relacionado con el bajo acceso a la seguridad social para la población ocupada, por estar en la informalidad, que en México representa cerca de 60 por ciento (INEGI, 2019), o bien a bajos niveles de población adulta mayor que cuentan con pensiones contributivas, cerca de 20 por ciento (Damián, 2016). Por otra parte, también se hace referencia a la debilitadas y fragmentadas instituciones del Estado (Araujo González, 2015). En México, el sistema de salud se encuentra fragmentado pues el acceso depende de la situación socioeconómica y laboral de la población, así como una marcada diferenciación en el acceso y calidad de 
los servicios de salud; además, presentan debilidad financiera esto en parte a un bajo gasto del gobierno en salud en términos per cápita y en relación al PIB (Martínez y Murayama, 2016). En este contexto, la pandemia del Covid-19 ha revelado o confirmado que los sistemas sanitarios de la región, incluido el de México, no estaban preparados para hacer frente al reto que impone la misma pandemia, evidenciando la crisis de los sistemas de salud (Huenchuan, 2020b). La falta de respuesta de los sistemas de salud representa un factor que puede empeorar la situación de vulnerabilidad de los adultos mayores (Huenchuan, 2020a).

Aunque la interacción social se ha documentado como un factor de contagio del Covid-19, en esta sección retomamos el elemento social y familiar para examinar su asociación con la vulnerabilidad familiar, examinando el tipo de arreglo familiar de los adultos mayores y la ayuda familiar recibida, entre otras características familiares.

\section{Metodología}

En este manuscrito utilizamos dos fuentes de información con el propósito de estimar los factores de riesgo asociados a dar positivo a Covid-19, ser hospitalizado o intubado; y para analizar la vulnerabilidad socioeconómica de las personas adultas mayores en México.

Combinar diferentes fuentes de información es bastante común y es muy utilizado por las ventajas que ofrece. Por ejemplo, permite extender la utilidad de cada fuente de información más allá de los propósitos inmediatos para los que fueron creadas (Raghunathan \& Arbor, 2008). En este caso, se busca utilizar datos sobre Covid-19 de una base de datos para calcular una serie de índices de riesgo, y dichos índices después se calculan usando otra base de datos con información adicional, la cual se usa para contextualizar dichos riesgos.

\section{Fuentes de información}

Para estimar los factores de riesgo asociados a positividad, hospitalización e intubación por Covid-19 y crear los índices de riesgo respectivos, se utiliza información de la base de datos de Covid-19 que elabora la Dirección General de Epidemiología de la Secretaría de Salud. Para este estudio se hizo el corte el 7 de septiembre de 2020 y se seleccionó a la población de 50 años o más a nivel nacional. El total de registros a esa fecha fue de 340,968 , que incluye a todos los individuos que se han realizado una prueba de Covid-19 y sus diferentes resultados: los negativos, los positivos, los hospitalizados, intubados y/o los fallecidos. Además, la base de datos con- 
tiene información sobre algunas características demográficas, enfermedades crónicas, factores de riesgo como tabaquismo y obesidad, entre otras.

El Estudio Nacional de Salud y Envejecimiento en México (ENASEM), es la segunda fuente de información utilizada. En esta base de datos se cuenta con la información utilizada en la base de datos Covid-19. Con dichos datos se calculan los índices de riesgo para cada sujeto en ENASEM, y se identifica a aquellos que tienen un riesgo alto de presentar Covid-19. Enseguida se analizan algunas características de la vulnerabilidad socioeconómica y familiar de la población adulta mayor con altos riegos de Covid-19.

El ENASEM es un estudio longitudinal, con representatividad nacional y urbano/rural de la población de 50 años o más en México. Iniciado desde 2001, el estudio tiene como objetivo examinar el proceso de envejecimiento en México pues cuenta con información relacionada a salud, cargas de enfermedades y discapacidades, así como con una amplia perspectiva sociodemográfica, económica y familiar (Wong, Michaels-Obregon and Pa1loni, 2017). La línea base del levantamiento se llevó a cabo en el año 2001 (nacidos hasta 1951), con rondas de seguimiento en los años de 2003, 2012, 2015 y 2018, pero manteniendo representatividad de la población de 50 años o más en las rondas de 2012 (nacidos entre 1952 y 1961) y 2018 (nacidos entre 1962 y 1967). Para el propósito de esta investigación tomamos la quinta y más reciente ronda de información, la de 2018 que cuenta con 13,316 registros de población de 50 años o más, que completaron una entrevista directa y tienen información completa para las variables edad, sexo, hipertensión, diabetes e índice de masa corporal.

\section{Métodos y construcción de variables}

Con los datos de la base Covid-19 se estimaron tres modelos de regresión logística, a) el de positividad a Covid-19, b) el de ser hospitalizado entre los positivos, y c) el de ser intubado entre los positivos hospitalizados; las tres variables dependientes con código 1 si la condición está presente y código 0 sí está ausente. Las variables independientes que se incluyen en cada uno de los modelos son: sexo (hombre, mujer), edad quinquenal en grupos (50 a 54, 55 a 59, 60 a 64, 65 a 69, 70 a 74, 75 a 79, 80 a 84, 85 o más), la presencia por separado de cada una de hipertensión, diabetes, y obesidad (si, no).

Se utilizó la metodología de Sullivan ((Sullivan, Massaro and D'Agostino, 2004; Mehta et al., 2016) para elaborar un índice basado en puntuaciones, en lugar de usar coeficientes de regresión, esto con la finalidad 
de tener estimaciones numéricas menos complicadas. Se procedió de la siguiente manera: una vez que se estimaron los modelos de regresión, en cada uno de ellos se identificó a la categoría que tenía el coeficiente con menor valor, después se dividió cada uno de los coeficientes de las variables independientes entre el coeficiente de la categoría con menor valor y se redondeó al entero más cercano. Hacerlo de esta manera permite relativizar la contribución de cada categoría a la positividad a Covid-19, ser hospitalizado o intubado. A las puntuaciones obtenidas las denominamos "coeficientes ponderados".

Estos coeficientes se sustituyen en las variables sexo, edad quinquenal, diabetes, hipertensión y obesidad de la base de datos ENASEM y para cada individuo se calcula el riesgo estimado de dar positivo, ser hospitalizado o intubado por Covid-19. Después se divide a la muestra ENASEM por cuartiles de menor a mayor, y se hace así para cada uno de los tres indicadores de riesgo. Finalmente, para la población de 50 años o más, utilizando la información de ENASEM, se hace un análisis descriptivo de las características socioeconómicas y de los recursos familiares con que cuentan las personas adultas mayores que tienen un riesgo alto, definido como estar en el cuartil más alto de riesgo de ser positivos/hospitalizados/intubados por Covid-19, respectivamente.

Lo anterior justifica la selección de la ENASEM pues tiene todas las variables que se utilizan en la base de datos de Covid-19 para estimar los modelos de regresión y los índices mencionados. Pero además es una fuente de información muy completa, que permite calcular los índices de riesgo de Covid-19, y analizarla por diferentes características socioeconómicas y familiares del grupo de personas adultas mayores.

\section{Adultos Mayores en Riesgo de Covid-19}

En cuanto a la pertinencia del uso de dos bases de datos para abordar la vulnerabilidad socioeconómica y familiar ante el Covid-19, lo primero que se presenta es una comparación de la distribución de la información en las dos bases de datos (Ver Tabla 1). El porcentaje de mujeres en la base de Covid-19 es de 53 por ciento, mientras que en la de ENASEM 2018 es 57 por ciento; la estructura por edad es muy similar entre ambas fuentes de información, los porcentajes de hipertensión y obesidad son ligeramente más altos en ENASEM (39.8 y 28.0) en comparación con la base de Covid-19 (37.0 y 19.3), mientras que el porcentaje que reporta diabetes es más alto en la base Covid-19 (29.0 por ciento) que en ENASEM (22.5 por ciento). 
Adultos mayores en riesgo de Covid-19 y sus vulnerabilidades socieconómicas y familiares ... / C. GONZÁLEZ GONZÁLEZ et al.

Tabla 1. Características de la población de 50 años o más

\begin{tabular}{lrrrrr}
\hline & Covid-19 & ENASEM_18 & & Covid-19 & ENASEM_18 \\
\hline Sexo & $(\mathrm{n}=340,968)$ & $(\mathrm{n}=14,900)$ & Hipertensión & $(\mathrm{n}=340,968)$ & $(\mathrm{n}=14,884)$ \\
Hombres & 47.0 & 43.0 & $\mathrm{No}$ & 63.0 & 60.2 \\
Mujeres & 53.0 & 57.0 & $\mathrm{Si}$ & 37.0 & 39.8 \\
Edad & $(\mathrm{n}=340,968)$ & $(\mathrm{n}=14,900)$ & Diabetes & $(\mathrm{n}=340,968)$ & $(\mathrm{n}=14,872)$ \\
$50-54$ & 27.2 & 26.0 & $\mathrm{No}$ & 71.0 & 77.5 \\
$55-59$ & 22.3 & 21.8 & $\mathrm{Si}$ & 29.0 & 22.5 \\
$60-64$ & 16.6 & 16.5 & Obesidad & $(\mathrm{n}=340,968)$ & $(\mathrm{n}=13,344)$ \\
$65-69$ & 12.1 & 13.1 & $\mathrm{No}$ & 80.7 & 72.0 \\
$70-74$ & 8.7 & 9.3 & $\mathrm{Si}$ & 19.3 & 28.0 \\
$75-79$ & 6.0 & 6.5 & & & \\
$80-84$ & 3.9 & 3.8 & & & \\
$85-$-más & 3.2 & 2.9 & & & \\
\hline
\end{tabular}

Fuente: elaboración propia con base en datos de la base Covid-19 de la Secretaria de Salud con corte al 7 de septiembre de 2020, y la quinta ronda del Estudio Nacional de Salud y Envejecimiento en México (ENASEM), datos ponderados de 2018.

\section{El Covid-19 en la población de 50 años o más en México (al 7 de septiembre de 2020)}

Hablar del riesgo de Covid-19 en adultos mayores en México y a nivel mundial es relativo, pues depende del nivel de pruebas que se están realizando en el país durante las diversas fases de la pandemia. Según la Universidad Johns Hopkins (Dowdy y D'Soulza, 2020), el umbral para que el porcentaje de positividad sea demasiado alto es de cinco por ciento. En julio de 2020 algunos países como Australia, Corea del Sur y Uruguay estaban muy por debajo del umbral de cinco por ciento, mientras que otros países como México y Nigeria tenían niveles muy por encima de ese nivel. Esto se debe en parte a otro indicador, el número de pruebas por habitantes. Los lineamientos de la vigilancia epidemiológica de Covid-19 en México señalan que solamente se harán pruebas en pacientes graves (Secretaría de Salud, 2020) y eso incrementa la positividad.

De acuerdo con la base de datos de la Secretaría de Salud, hasta el 7 de septiembre del 2020, el porcentaje de población de 50 años o más en México que dio positivo a Covid-19 es de 69.4 por ciento (ver Tabla 2). La distribución fue: 37.8 por ciento dio positivo con un manejo ambulatorio de la enfermedad, 27.4 por ciento requirió ser hospitalizado sin ser intuba- 
do y 4.2 por ciento requirió intubación.

Tabla 2. Resultados de la prueba PCR al Covid-19 de la población de 50 años o más en México al 7 de septiembre de 2020

\begin{tabular}{lrrr}
\hline Resultados del examen & Frecuencia & Porcentaje & \% fallecido \\
\hline Negativo & 104,325 & 30.6 & 8.1 \\
Positivo ambulatorio & 128,790 & 37.8 & 4.8 \\
Positivo hospitalizado & 93,471 & 27.4 & 41.0 \\
Positivo hospitalizado e intubado & 14,382 & 4.2 & 75.4 \\
Total & 340,968 & 100.0 & 18.7 \\
\hline
\end{tabular}

Fuente: elaboración propia con base en datos de la Secretaria de Salud, corte al 7 de septiembre de 2020.

Además, en México las pruebas están ligadas a una situación crítica de salud y quienes acuden a realizársela aunque resulten negativos a Covid-19 pueden presentar complicaciones. Por ejemplo, 8.1 por ciento de los que resultaron negativos a la prueba de Covid-19 fallecieron, lo mismo que 4.8 por ciento de los positivos ambulatorios, y este porcentaje aumenta drásticamente entre los hospitalizados y los intubados, pues cuatro de cada diez de los hospitalizados y tres de cada cuatro intubados fallecieron.

\section{Estimación de los riesgos de dar positivo, ser hospitalizado e intubado}

Con base en las pruebas reportadas por la Secretaría de Salud al 7 de septiembre $(n=340,968)$, se estimaron los coeficientes de regresión logística y las Razones de Momios (RM) para dar positivo al Covid-19, ser hospitalizado e intubado (Ver Tabla 3). Los resultados muestran que la edad es uno de los principales factores para resultar positivo a Covid-19, los que tienen entre 70 y 74 años presentan la mayor razón de momios $(\mathrm{RM}=1.441)$; ser hombre $(\mathrm{RM}=1.271)$, tener diabetes $(\mathrm{RM}=1.260)$, tener obesidad $(\mathrm{RM}=1.255)$ o tener hipertensión $(\mathrm{RM}=1.093)$ también incrementan la probabilidad de dar positivo a Covid-19.

Entre los que dieron positivo a Covid-19 $(\mathrm{n}=236,643)$ se estimó el efecto de ser hospitalizado, la edad tiene el efecto más importante para ser hospitalizado y el riesgo se incrementa de manera constante, en comparación con una persona de 50 a 54 años, la razón de momios de ser hospitalizado para una persona de 85 años o más es 4.526 . 
Adultos mayores en riesgo de Covid-19 y sus vulnerabilidades socieconómicas y familiares ... / C. GONZÁLEZ GONZÁLEZ et al.

Tabla 3: Modelos de regresión logística de dar positivo, ser hospitalizado o intubado de la población de 50 años o más en México, al 7 de septiembre de 2020

\begin{tabular}{|c|c|c|c|c|c|c|c|c|c|}
\hline \multirow[b]{2}{*}{ Características } & \multicolumn{3}{|c|}{ Positivo } & \multicolumn{3}{|c|}{ Hospitalizado } & \multicolumn{3}{|c|}{ Intubado } \\
\hline & Coef. & $\mathrm{RM}$ & Signif. & Coef. & $\mathrm{RM}$ & Signif. & Coef. & $\mathrm{RM}$ & Signif. \\
\hline \multicolumn{10}{|l|}{ Sexo } \\
\hline Hombres & 0.240 & 1.271 & 0.000 & 0.491 & 1.271 & 0.000 & 0.443 & 1.557 & 0.000 \\
\hline \multicolumn{10}{|l|}{ Mujeres (ref.) } \\
\hline \multicolumn{10}{|l|}{ Edad } \\
\hline \multicolumn{10}{|l|}{ 50-54 (ref.) } \\
\hline $55-59$ & 0.106 & 1.112 & 0.000 & 0.305 & 1.112 & 0.000 & 0.341 & 1.406 & 0.000 \\
\hline $60-64$ & 0.222 & 1.249 & 0.000 & 0.667 & 1.249 & 0.000 & 0.624 & 1.866 & 0.000 \\
\hline $65-69$ & 0.340 & 1.405 & 0.000 & 0.983 & 1.405 & 0.000 & 0.822 & 2.274 & 0.000 \\
\hline $70-74$ & 0.366 & 1.441 & 0.000 & 1.233 & 1.441 & 0.000 & 0.894 & 2.446 & 0.000 \\
\hline $75-79$ & 0.348 & 1.417 & 0.000 & 1.411 & 1.417 & 0.000 & 0.936 & 2.551 & 0.000 \\
\hline $80-84$ & 0.249 & 1.286 & 0.000 & 1.537 & 1.282 & 0.000 & 0.871 & 2.389 & 0.000 \\
\hline 85 o más & 0.035 & 1.036 & 0.111 & 1.510 & 1.036 & 0.111 & 0.706 & 2.026 & 0.000 \\
\hline \multicolumn{10}{|l|}{ Hipertensión } \\
\hline \multicolumn{10}{|l|}{ No (ref.) } \\
\hline $\mathrm{Si}$ & 0.089 & 1.093 & 0.000 & 0.219 & 1.093 & 0.000 & 0.113 & 1.119 & 0.000 \\
\hline \multicolumn{10}{|l|}{ Diabetes } \\
\hline \multicolumn{10}{|l|}{ No (ref.) } \\
\hline $\mathrm{Si}$ & 0.231 & 1.260 & 0.000 & 0.550 & 1.260 & 0.000 & 0.273 & 1.313 & 0.000 \\
\hline \multicolumn{10}{|l|}{ Obesidad } \\
\hline \multicolumn{10}{|l|}{ No (ref.) } \\
\hline $\mathrm{Si}$ & 0.228 & 1.255 & 0.000 & 0.276 & 1.255 & 0.000 & 0.343 & 1.409 & 0.000 \\
\hline Constante & 0.396 & 1.485 & 0.000 & -1.392 & 1.485 & 0.000 & -3.766 & 0.023 & 0.000 \\
\hline
\end{tabular}

Fuente: elaboración propia con base en datos de la Secretaría de Salud, corte al 7 de septiembre del 2020 .

Los hombres tienen 63.4 por ciento más probabilidad de ser hospitalizados que las mujeres, los que tienen diabetes tienen un RM de 1.733, para quienes presentan obesidad el RM es 1.317 y para quienes tienen hipertensión el RM es 1.244.

Entre los que fueron hospitalizados al dar positivo a Covid-19 $(\mathrm{n}=107,853)$, se corrió el modelo de regresión logística de ser intubado o no. Los resultados señalan que ser intubado presenta relación con el sexo, la edad, las enfermedades crónicas y obesidad. Los que tienen entre 75 y 79 años y que resultaron positivos a Covid-19 son el grupo que tiene la 
más alta probabilidad de ser intubados con una RM de 2.551, los hombres tienen 55.7 por ciento más probabilidad de ser intubado que las mujeres, la obesidad que en los modelos de positivo y hospitalizado tenía una RM menor que hipertensión y diabetes para este caso presenta una RM de 1.409, mientras que para diabetes la RM es de 1.313 y de 1.119 para hipertensión.

En los tres modelos, la edad es el factor más importante; sin embargo, en el caso de hospitalización tener diabetes también se presenta como un factor importante, así como la obesidad en el caso de ser intubado.

\section{Construcción de índices de riesgo}

Los índices de riesgo se crearon basados en puntuaciones que derivan de los coeficientes de los modelos de regresión. En la regresión de ser positivo a Covid-19, el grupo de edad 85 años o más es el que tuvo el coeficiente más bajo en el modelo de regresión, en los modelos de hospitalizado e intubado fue la variable hipertensión, y estás categorías recibieron un puntaje de 1 , y las categorías de referencia un puntaje de 0 . Las puntuaciones para el resto de las categorías se estimaron a partir de la división de cada uno de los coeficientes respecto al coeficiente más bajo en cada modelo de regresión. De esta manera, el índice de positivo a Covid-19 va de 0 a 34 puntos, el de hospitalizado de 0 a 14 y el de intubado de 0 a 18, estos de acuerdo con la suma de los puntajes más altos en cada característica observada y que aparecen en negritas en la Tabla 4.

En la base de datos de ENASEM se sustituyeron las puntuaciones obtenidas, de tal manera que cada participante de 50 años o más, con información completa para las variables edad, sexo, hipertensión, diabetes e índice de masa corporal, tiene una puntuación de riesgo para dar positivo, ser hospitalizado y ser intubado acorde a los resultados de los modelos de regresión estimados en la base de datos Covid-19. Lo siguiente fue dividir a la población por cuartiles en los tres índices de riesgo analizados. Así junto con el índice recién estimado y las características socioeconómicas y familiares con que cuenta el ENASEM se pueden explorar las vulnerabilidades de los adultos mayores en caso de dar positivos a Covid-19, ser hospitalizado o ser intubado. 
Adultos mayores en riesgo de Covid-19 y sus vulnerabilidades socieconómicas y familiares ... / C. GONZÁLEZ GONZÁLEZ et al.

Tabla 4: Puntuaciones para el índice de dar positivo, ser hospitalizado o intubado de la población de 50 años o más en México, al 7 de septiembre de 2020

\begin{tabular}{|c|c|c|c|}
\hline \multirow[b]{2}{*}{ Características } & \multicolumn{3}{|c|}{ Puntuación para el índice } \\
\hline & Positivo & Hospitalizado & Intubado \\
\hline \multicolumn{4}{|l|}{ Sexo } \\
\hline Hombres & 7 & 2 & 4 \\
\hline Mujeres (ref.) & 0 & 0 & 0 \\
\hline \multicolumn{4}{|l|}{ Edad } \\
\hline 50-54 (ref.) & 0 & 0 & 0 \\
\hline $55-59$ & 3 & 1 & 3 \\
\hline $60-64$ & 6 & 3 & 6 \\
\hline $65-69$ & 10 & 4 & 7 \\
\hline $70-74$ & 10 & 6 & 8 \\
\hline $75-79$ & 10 & 6 & 8 \\
\hline $80-84$ & 7 & 7 & 8 \\
\hline 85 o más & 1 & 7 & 6 \\
\hline \multicolumn{4}{|l|}{ Hipertensión } \\
\hline No (ref.) & 0 & 0 & 0 \\
\hline $\mathrm{Si}$ & 3 & 1 & 1 \\
\hline \multicolumn{4}{|l|}{ Diabetes } \\
\hline No (ref.) & 0 & 0 & 0 \\
\hline $\mathrm{Si}$ & 7 & 3 & 2 \\
\hline \multicolumn{4}{|l|}{ Obesidad } \\
\hline No (ref.) & 0 & 0 & 0 \\
\hline $\mathrm{Si}$ & 7 & 1 & 3 \\
\hline
\end{tabular}

Fuente: elaboración propia con base en datos de la Secretaría de Salud, corte al 7 de septiembre del 2020.

\section{Vulnerabilidades socioeconómicas y familiares de la población de 50 años o más}

El análisis de las vulnerabilidades socioeconómicas y familiares utiliza la información del ENASEM y se centra en 25 por ciento de la población que presenta el riesgo más alto (cuartil 4 del índice de riesgo) de presentar una de las condiciones asociadas a la enfermedad de Covid-19. Se describen sus características socioeconómicas y familiares con el objetivo de identificar a los vulnerables entre los vulnerables. Es necesario mencionar que 
se está considerando en el análisis a los que tienen los puntajes o el nivel de riesgo más alto a la enfermedad del Covid-19 en cada uno de los tres índices construidos; y no se está calculando directamente la probabilidad de dar positivo, ser hospitalizado o intubado por Covid-19, son dos conceptos completamente diferentes. En dado caso, esto sería un futuro tema de investigación dada la oportunidad que brinda el ENASEM por ser un panel longitudinal.

En sí, las personas adultas mayores representan un grupo socioeconómicamente vulnerable; sin embargo, en el cuartil más alto de riesgo de enfermar de Covid-19 es posible observar algunas características que lo define como más vulnerable.

Un primer resultado es que el cuartil más alto, aquellos que presentan riesgo alto de dar positivo a Covid-19 está compuesto mayoritariamente por hombres, que representan 82.2 por ciento y entre los que tienen 65 años o más con 73.8 por ciento, ambos porcentajes resultan superiores a los observados en el total de adultos mayores que es de 44.9 por ciento (Tabla $5)$. Dentro de las características socioeconómicas, una proporción mayor de los del cuartil de riesgo más alto tiene una escolaridad de 6 o menos años respecto al total de adultos mayores (68 por ciento versus 55.7 por ciento); en cuanto a su actividad económica se observa que 27.5 por ciento realiza su trabajo sin disponer de un local, es decir, como ambulantes, en puestos improvisados, en vehículos o puestos semifijos, lo que los vuelve vulnerables a la exposición del Covid-19 ante la falta de servicios sanitarios durante su jornada laboral, y este porcentaje es ligeramente superior al total el cual es de 25.4 por ciento; además, 25.1 por ciento no labora por ser mayor de edad, estar enfermo, porque no tienen clientes o no encuentran trabajo, mientras que en la población total es de 16.9 por ciento.

Siete de cada 10 personas adultas mayores considera que su situación económica es regular o mala, prácticamente no hay diferencias entre los del cuartil más alto y la población en general. Otra característica para destacar respecto a la población vulnerable es que un porcentaje mayor de los ubicados en el cuartil más alto de riesgo a dar positivo, reciben transferencias gubernamentales. En cuanto a la vulnerabilidad familiar, de los que están en el riesgo más alto de dar positivo a Covid-19, 4.5 por ciento de la población de 50 años o más tiene menos de dos hijos vivos, 9.3 por ciento vive en hogares unipersonales, 32.6 por ciento recibe ayuda económica y 41.2 por ciento recibe ayuda no económica.

En cuanto a los que tienen un riesgo alto de dar positivo y ser hospitalizados, 67.6 por ciento son hombres y 86.5 por ciento tiene 65 o más 
años; además, 72.9 por ciento tienen seis o menos años de estudios. En cuanto a la actividad económica, uno de cada tres no labora porque tienen edad avanzada, están enfermos, porque no tienen clientes o no encuentran trabajos; y 72.8 por ciento considera que su situación económica es regular o mala, y 40.5 por ciento recibe transferencias gubernamentales. Como reflejo del respaldo familiar, se observa que 36.7 por ciento recibe ayuda económica familiar, porcentaje superior al total; además, tienen un mayor número de hijos vivos, pues 28.1 por ciento tiene siete o más hijos. Este grupo de adultos mayores se destaca del conjunto de adultos mayores por vivir en hogar unipersonal o solo con su pareja (Tabla 5). Además, destaca que 13.2 por ciento no tiene escolaridad, 12 por ciento en algún momento de los últimos dos años no tuvieron dinero suficiente para comida, 72.2 por ciento reporta una situación regular o mala, 93.6 por ciento no tiene ahorros, y 61.5 por ciento no recibe transferencias gubernamentales.

En cuanto a los que tienen riesgo alto de ser intubados, 84.4 por ciento son hombres, 13.7 por ciento no tienen escolaridad, 10.8 por ciento en los últimos dos años en algún momento no tenía dinero para comer y se fue a dormir sin comer, además 71.5 por ciento reporta su situación económica como regular o mala, 75.5 por ciento está pagando su casa, 51.1 por ciento no recibe una pensión contributiva, 92.9 por ciento no tiene ahorro, $\mathrm{y}$ 64.9 por ciento no recibe transferencias gubernamentales. En cuanto a la vulnerabilidad familiar, 3.3 por ciento no tienen hijos, 12.2 por ciento viven solos, 33.2 por ciento recibe ayuda económica, 39.9 por ciento recibe ayuda no económica.

Los resultados señalan que entre los que tienen un riesgo alto - de dar positivo a Covid-19, ser hospitalizados o ser intubados- están presentes una serie de características que los hacen vulnerables a la enfermedad. La situación económica y/o familiar en un gran porcentaje de individuos es precaria, y de materializarse el riesgo se pondría en serios predicamentos su bienestar general.

\section{DisCUSIÓN Y CONCLUSIONES}

Las vulnerabilidades son de diversa índole, pueden ser físicas, emocionales, cognitivas o financieras, y quienes presentan vulnerabilidad en cualquiera de estas dimensiones de la vida corren un mayor riesgo. La pandemia del Covid-19 ha trastocado la vida de todos los habitantes del mundo, pero esos cambios se exacerban en los grupos poblacionales que presentan mayor riesgo de contagiarse por esta enfermedad. 
Tabla 5: Distribución porcentual de la población de 50 años o más, total y los ubicados en el nivel más alto de riesgo, ENASEM 2018

\begin{tabular}{|c|c|c|c|c|}
\hline Características & $\begin{array}{l}\text { Positivos } \\
\text { Cuartil } 4\end{array}$ & $\begin{array}{r}\text { Hospitalizados } \\
\text { Cuartil } 4\end{array}$ & $\begin{array}{r}\text { Intubados } \\
\text { Cuartil } 4\end{array}$ & Total \\
\hline \multicolumn{5}{|l|}{ Sexo } \\
\hline Mujer & 17.8 & 32.4 & 15.6 & 55.1 \\
\hline Hombres & 82.2 & 67.6 & 84.4 & 44.9 \\
\hline \multicolumn{5}{|l|}{ Edad } \\
\hline $50-54$ & 4.9 & 0.0 & 0.0 & 26.8 \\
\hline $55-59$ & 5.5 & 2.8 & 3.6 & 22.4 \\
\hline $60-64$ & 15.8 & 10.7 & 18.2 & 16.7 \\
\hline $65-69$ & 26.8 & 15.6 & 21.3 & 12.9 \\
\hline $70-74$ & 21.8 & 28.3 & 26.9 & 8.9 \\
\hline $75-79$ & 15.8 & 19.1 & 17.9 & 6.3 \\
\hline $80-84$ & 8.6 & 13.7 & 10.2 & 3.5 \\
\hline 85 o más & 0.7 & 9.8 & 1.8 & 2.5 \\
\hline \multicolumn{5}{|l|}{ Escolaridad } \\
\hline 0 & 13.2 & 17.7 & 13.7 & 10.2 \\
\hline 1 a 5 & 31.1 & 33.1 & 30.3 & 23.1 \\
\hline 6 & 23.8 & 22.0 & 23.6 & 22.4 \\
\hline 7 a 9 & 14.2 & 14.0 & 14.6 & 24.3 \\
\hline 10 a 12 & 5.1 & 5.0 & 5.9 & 8.1 \\
\hline 13 o más & 12.7 & 8.1 & 11.8 & 12.0 \\
\hline \multicolumn{5}{|l|}{ Dinero para comida } \\
\hline $\mathrm{Si}$ & 70.9 & 72.2 & 73.8 & 70.8 \\
\hline No, pero con comida & 17.2 & 17.2 & 15.4 & 17.0 \\
\hline No, sin comer & 12.0 & 10.6 & 10.8 & 12.2 \\
\hline \multicolumn{5}{|l|}{ Actividad Económica } \\
\hline Trabaja sin local & 27.5 & 19.3 & 24.9 & 25.4 \\
\hline Trabaja con local & 16.3 & 9.6 & 15.6 & 23.5 \\
\hline Quehacer del hogar & 9.6 & 13.9 & 8.0 & 23.6 \\
\hline Pensionado & 21.5 & 22.5 & 23.5 & 10.6 \\
\hline Otra & 25.1 & 34.7 & 28.1 & 16.9 \\
\hline \multicolumn{5}{|l|}{ Situación económica } \\
\hline Excelente, muy buena y buena & 27.8 & 27.3 & 28.5 & 29.4 \\
\hline Regular y mala & 72.2 & 72.8 & 71.5 & 70.6 \\
\hline
\end{tabular}

Fuente: elaboración propia con la base de datos Covid-19 de la Secretaria de Salud con corte al 7 de septiembre de 2020, y de la quinta ronda del Estudio Nacional de Salud y Envejecimiento en México (ENASEM), datos ponderados de 2018. 
Adultos mayores en riesgo de Covid-19 y sus vulnerabilidades socieconómicas y familiares ... / C. GONZÁLEZ GONZÁLEZ et al.

Tabla 5: Continuación

\begin{tabular}{|c|c|c|c|c|}
\hline Características & $\begin{array}{l}\text { Positivos } \\
\text { Cuartil } 4\end{array}$ & $\begin{array}{r}\text { Hospitalizados } \\
\text { Cuartil } 4 \\
\end{array}$ & $\begin{array}{r}\text { Intubados } \\
\text { Cuartil } 4\end{array}$ & Total \\
\hline \multicolumn{5}{|l|}{ Tenencia de la vivienda } \\
\hline Renta & 3.8 & 3.4 & 3.4 & 5.0 \\
\hline Pagada & 75.3 & 75.7 & 75.5 & 69.9 \\
\hline Pagando & 20.8 & 21.0 & 21.1 & 25.2 \\
\hline \multicolumn{5}{|l|}{ Seguro médico } \\
\hline No & 7.6 & 8.0 & 8.1 & 11.1 \\
\hline Derechohabiencia & 58.0 & 63.7 & 63.9 & 55.5 \\
\hline Seguro Popular & 34.4 & 28.2 & 28.1 & 33.4 \\
\hline \multicolumn{5}{|l|}{ Ahorro } \\
\hline No & 93.6 & 93.3 & 92.9 & 93.8 \\
\hline $\mathrm{Si}$ & 6.4 & 6.7 & 7.1 & 6.2 \\
\hline \multicolumn{5}{|l|}{ Transf. Gubernamentales } \\
\hline No & 61.5 & 59.5 & 64.9 & 73.0 \\
\hline $\mathrm{Si}$ & 38.5 & 40.5 & 35.1 & 27.0 \\
\hline \multicolumn{5}{|l|}{ Hijos } \\
\hline 0 a 1 & 4.5 & 4.1 & 3.3 & 6.5 \\
\hline 2 a 3 & 34.2 & 30.4 & 36.0 & 44.8 \\
\hline 4 a 6 & 38.7 & 37.4 & 38.0 & 35.4 \\
\hline 7 o más & 22.6 & 28.1 & 22.8 & 13.4 \\
\hline \multicolumn{5}{|l|}{ Ayuda económica recibida } \\
\hline No & 67.4 & 63.3 & 66.8 & 72.4 \\
\hline $\mathrm{Si}$ & 32.6 & 36.7 & 33.2 & 27.6 \\
\hline \multicolumn{5}{|c|}{ Ayuda no económica recibida } \\
\hline No & 58.8 & 57.4 & 60.1 & 56.7 \\
\hline $\mathrm{Si}$ & 41.2 & 42.6 & 39.9 & 43.4 \\
\hline \multicolumn{5}{|l|}{ Arreglo familiar } \\
\hline Unipersonal & 9.3 & 11.8 & 8.9 & 9.6 \\
\hline Pareja & 25.9 & 24.8 & 28.9 & 20.2 \\
\hline AM con hijos & 30.4 & 29.2 & 29.7 & 35.2 \\
\hline AM, hijos, nietos & 10.6 & 12.0 & 11.3 & 11.4 \\
\hline Otros extensos y com. & 23.8 & 22.3 & 21.2 & 23.7 \\
\hline
\end{tabular}

Fuente: elaboración propia con la base de datos Covid-19 de la Secretaria de Salud con corte al 7 de septiembre de 2020, y de la quinta ronda del Estudio Nacional de Salud y Envejecimiento en México (ENASEM), datos ponderados de 2018. 
Sin duda existe un riesgo y se incrementaría la vulnerabilidad de las personas si se es positivo, se hospitaliza o si tiene que ser intubado. Identificar por cuartiles de riesgo a la población que pudiera estar expuesta a Covid-19 y describir las características socioeconómicas y familiares brinda la posibilidad de discutir las acciones que se deberán tomar para minimizar el riesgo y apoyar a aquellos que están en vulnerabilidad.

Justo en ese sentido, tal y como sucede en otras latitudes, la edad y ser hombre están relacionados con mayor probabilidad de resultar positivo a Covid-19 (Aquino-Camchari, Quispe-Arrieta and Hueman Costillo, 2020; Bonanad et al., 2020; Huenchuan, 2020a). En los tres indicadores analizados el grupo de alto riesgo está compuesto mayoritariamente por hombres, y uno de cada cuatro tiene 80 años o más.

A medida que las consecuencias del Covid-19 se presentan en la sociedad, se espera que los recursos de las Personas Adultas Mayores se vean impactados (Li and Mutchler, 2020), directa e indirectamente. De manera directa, en los grupos de riesgo alto, en México siete de cada diez reportan una situación económica regular o mala, aunque esto prevalece en la población en general. Por ejemplo, si llegan a ser hospitalizados o intubados sin duda su situación económica se volverá más precaria, particularmente para aquellos que no tienen acceso a los servicios de salud que representan ocho por ciento. Además, Calderón-Larrañaga et al. (2020) señalan que las personas mayores de entornos de bajos ingresos tienen más probabilidades de sufrir afecciones crónicas, multimorbilidad o incluso fragilidad, y que por lo tanto también tienen un mayor riesgo de contraer enfermedades asociadas a Covid-19, y por lo tanto de ser económicamente más vulnerables.

Indirectamente el Covid-19 está afectando a toda la población de 50 años o más en México, está deteriorando la ya precaria situación económica de muchos adultos mayores que han tenido que dejar de trabajar, trabajar menos horas o incluso afectado su ingreso por transferencias familiares ya que sus hijos también están resintiendo los efectos de la pandemia. Entonces, a medida que se expresen las consecuencias económicas derivadas de la crisis del Covid-19, se espera que los recursos económicos de las personas adultas mayores se vean afectados. Los efectos se darán con más fuerza en las zonas pobres, donde la seguridad económica ya es precaria para muchas personas mayores (Li and Mutchler, 2020; Mohanty, 2020; Nicola et al., 2020). Estos resultados ponen de manifiesto las implicaciones de la actual pandemia y los retos futuros para proveer seguridad económica al grupo de adultos mayores, en donde los recursos económicos $\mathrm{y}$ familiares juegan un papel importante. 
Las limitaciones del estudio están relacionadas con la falta de información sobre el conjunto de factores asociados a contagiarse y agravamiento del Covid-19, ya que no se incluyen los factores de interacción social en el riesgo de dar positivo al Covid-19. Sin embargo, se incluyen los factores de agravamiento del Covid-19 como la obesidad y las enfermedades crónicas (hipertensión y diabetes) para identificar el riesgo de dar positivo, de ser hospitalizado o intubado.

Generar conocimiento sobre las condiciones de población vulnerable es uno de los objetivos de este manuscrito, evitar que las personas se vean afectadas por la pandemia del Covid-19 debe ser la prioridad de las políticas públicas. Es necesario desarrollar estrategias y apoyos encaminados a minimizar los impactos de eventos como el de la pandemia, asegurar el acceso a servicios de salud de calidad y oportunos, un ingreso económico acorde con las necesidades, y el mantenimiento de los estándares de bienestar de las personas adultas mayores. Además, la pandemia no solo está amenazando la vida de las personas adultas mayores, también está amenazando sus redes familiares y sociales, su empleo y su seguridad económica.

Aún y cuando no lleguen a ser contagiados por Covid-19, una parte importante de las personas adultas mayores habrá visto mermadas sus condiciones económicas, de salud y familiares. Lo reportado en este documento permite identificar las características que incrementan su vulnerabilidad, es necesario pensar a corto y mediano plazo, aunque parece que habrá una vacuna para todos en los siguientes meses, habrá que considerar los años de recuperación post-pandemia. México enfrentará una de las recesiones económicas más severas de su historia, con una significativa reducción en el bienestar general de la población y de las personas de 60 años o más.

Tal y cómo se menciona en el Policy Brief de las Naciones Unidas (2020), es necesario desarrollar acercamientos novedosos, respaldados por la evidencia y la información desagregada por edad, pero también por sexo y condiciones socioeconómicas relevantes. Consideramos que este estudio forma parte de ese cuerpo de evidencia que genera conocimiento sobre los efectos del Covid-19 en la población en edades avanzadas.

\section{Agradecimientos}

Los autores agradecen el apoyo del Sealy Center on Aging en la UTMB. El ENASEM está parcialmente financiado por el National Institutes of Health/National Institute on Aging (R01AG018016) en Estados Unidos y el Instituto Nacional de Estadística y Geografía (INEGI) en México. 


\section{REFERENCIAS BIBLIOGRÁFICAS}

Andrew, M. K. et al., 2012, "The impact of social vulnerability on the survival of the fittest older adults", in Age and Ageing, 41(2), pp. 161-165. doi: 10.1093/ ageing/afr176

Aquino-Camchari, C. R., Quispe-Arrieta, R. del C. and Hueman Costillo, K. M., 2020, "Covid-19 y su relación con poblaciones vulnerables", en Revista Habanera de Ciencias Medicas, 19(Suplemento), pp. 1-18.

Araujo González, R., 2015, "Vulnerabilidad y riesgo en salud: ¿dos conceptos concomitantes?", en Revista Novedades en Población, 11(21), pp. 89-96.

Avanoğlu Güler, A. and Akif Öztürk, M., 2020, "Covid-19 in chronic diseases”, in Gazi Medical Journal, 31(2), pp. 266-270. doi: 10.12996/gmj.2020.68

Bansal, M., 2020, "Cardiovascular disease and Covid-19”, in Diabetes and Metabolic Syndrome: Clinical Research and Reviews. Elsevier Ltd, 14(3), pp. 247-250. doi: 10.1016/j.dsx.2020.03.013

Bonanad, Clara et al., 2020, "Coronavirus: the geriatric emergency of 2020. Joint document of the section on geriatric cardiology of the Spanish society of cardiology and the Spanish society of geriatrics and gerontology", en Revista Espanola de Cardiología, 73(7), pp. 569-576. doi: 10.1016/j.recesp.2020.03.027

Calderón-Larrañaga, A. et al., 2020, “Covid-19: risk accumulation among biologically and socially vulnerable older populations", en Ageing Research Reviews, 63(July), pp. 1-5. doi: 10.1016/j.arr.2020.101149

CEPAL, 2020a, América Latina y el Caribe ante la pandemia del Covid-19: efectos económicos y sociales, Informe Especial Covid-19. 1. Santiago de Chile.

CEPAL, 2020b, El desafio social en tiempos del Covid-19, Informe especial Covid-19. 3. Santiago de Chile.

CEPAL, 2020c, Informe sobre impacto económico en América Latina y el Caribe de la enfermedad por coronavirus (Covid-19), Covid-19 Respuesta. Santiago de Chile.

Damián, A., 2016, “Seguridad social, pensiones y pobreza de los adultos mayores en México", en Acta Sociológica, 70, pp. 151-172. doi: 10.1016/j.acso.2017.01.007

Esteve, A. et al., 2020, "National age and coresidence patterns shape Covid-19 vulnerability", in Proceedings of the National Academy of Sciences of the United States of America, 117(28), pp. 16118-16120. doi: 10.1073/pnas.2008764117

Fenge, L. A. et al., 2012, "The impact of the economic recession on well-being and quality of life of older people", in Health and Social Care in the Community, 20(6), pp. 617-624. doi: 10.1111/j.1365-2524.2012.01077.x

Fernandes, K. T. et al., 2017, "Aging and individual vulnerability: a panorama of older adults attended by the family health strategy", en Texto and Contexto - Enfermagem, 26(2), pp. 1-10. doi: 10.1590/0104-07072017002700015 
Adultos mayores en riesgo de Covid-19 y sus vulnerabilidades socieconómicas y familiares ... / C. GONZÁLEZ GONZÁLEZ et al.

Gobierno de México, 2020, Covid-19 México, Conacyt, CentroGeo, GEoInt, DataLAb.

Guo, T. et al., 2020, "Clinical characteristics of elderly patients with Covid-19 in hunan province, China: a multicenter, retrospective study", in Gerontology, (66), pp. 467-475. doi: 10.1159/000508734

Huenchuan, S., 2020a, Covid-19: Recomendaciones generales para la atención a personas mayores desde una perspectiva de derechos humanos. Ciudad de México.

Huenchuan, S., 2020b, El derecho a la vida y la salud de las personas mayores en el marco de la pandemia por Covid-19. Ciudad de México.

Li, Y. and Mutchler, J. E., 2020, "Older adults and the economic impact of the Covid-19 pandemic", in Journal of Aging and Social Policy. Routledge, 32(4-5), pp. 477-487. doi: 10.1080/08959420.2020.1773191

Mallapaty, S., 2020, "The coronavirus is most deadly if you are older and male", in Nature, 585(3), pp. 16-17. doi: 10.1038/d41586-020-02483-2

Mehta, H. B. et al., 2016, "Regression coefficient-based scoring system should be used to assign weights to the risk index", en Journal of Clinical Epidemiology. Elsevier Inc, 79, pp. 22-28. doi: 10.1016/j.jclinepi.2016.03.031

Mohanty, S. K., 2020, “Contextualising geographical vulnerability to Covid-19 in India", en The Lancet Global Health. The Author(s). Published by Elsevier Ltd. This is an Open Access article under the CC BY 4.0 license, 8(9), pp. e1104e1105. doi: 10.1016/S2214-109X(20)30329-6

Nicola, M. et al., 2020, "The socio-economic implications of the coronavirus pandemic (Covid-19): A review”, in International Journal of Surgery. Elsevier, 78(March), pp. 185-193. doi: 10.1016/j.ijsu.2020.04.018

Ritchie, H. et al., 2020, Statistics and Research Coronavitus (Covid-19) Testing, Oxford Martian School. University of Oxford.

Rodríguez, M. and Wong, R., 2019, "Envejecimiento en México: obesidad”, en Boletín Informativo del ENASEM, (1), pp. 1-2.

Sullivan, L. M., Massaro, J. M. and D'Agostino, R. B., 2004, "Presentation of multivariate data for clinical use: the framingham study risk score functions", in Statistics in Medicine, 23(10), pp. 1631-1660. doi: 10.1002/sim.1742

Wong, R., Michaels-Obregon, A. and Palloni, A., 2017, "Cohort profile: the Mexican health and aging study (MHAS)", in International Journal of Epidemiology, 46(2), p. e2 (1-10). doi: 10.1093/ije/dyu263

\section{RESUMEN CURRICULAR DE LOS AUTORES}

César González González

Maestro en Demografía por el Colegio de la Frontera Norte y Doctor en Estudios de Población por el Colegio de México. Ha sido investigador en 
el Instituto Nacional de Geriatría (2009-2016) y actualmente es Profesor Investigador de la Facultad de Trabajo Social en la Universidad de Colima. Es miembro del Sistema Nacional de Investigadores (Nivel 1). Entre sus publicaciones recientes destacan "Impact of diabetes and disease duration on work status among US Older Adults" (Journal of Aging and Health, 2020), "Envejecimiento y mortalidad: condiciones de salud, económicas y familiares en el último año de vida de las personas mayores en México" (Papeles de Población, 2018) "Projecting diabetes prevalence among Mexicans aged 50 years and older: The Future Elderly Model-Mexico (FEM-Mexico)" (BMJ Open, 2017) y "Mortality inequality among older adults in Mexico: the combined role of infectious and chronic diseases" (Rev Panam Salud Publica, 2014).

Dirección electrónica: cgonzalez31@ucol.mx

Registro ORCID: https://orcid.org/0000-0001-7156-3220

\section{Karina Orozco Rocha}

Es Doctora en Estudios de Población por El Colegio de México. Economista y Maestra en Ciencias, área Finanzas por la Universidad de Colima. Sus líneas de investigación y publicaciones examinan el bienestar económico y las redes de protección en las edades avanzadas de los mexicanos. Su publicación más reciente es "Trabajo de cuidado en las fuentes de información estadística de México" (Realidad, Datos y Espacio, 2020) y "Recruitment and retention in aging research" (Encyclopedia of Gerontology and Population Aging, 2020). Pertenece al Sistema Nacional de Investigadores (SNI) del Consejo Nacional de Ciencia y Tecnología (CONACYT), Nivel 1. Actualmente es profesora en la Facultad de Economía de la Universidad de Colima.

Dirección electrónica: korozco9@ucol.mx

Registro ORCID: https://orcid.org/0000-0002-7748-6198

\section{Rafael Samper Ternent}

Médico Geriatra de la Pontificia Universidad Javeriana en Bogotá, Colombia y PhD de The University of Texas Medical Branch (UTMB) en Galveston, Estados Unidos. Es profesor Asistente en el Departamento de Medicina Interna de UTMB e Investigador del Sealy Center on Aging de la misma Universidad desde 2017. Desde 2009 es Co- Investigador de la Encuesta Nacional de Salud y Envejecimiento en México (ENASEM). Entre sus publicaciones recientes se destacan "Factors associated with pain at the end-of-life among older adults in Mexico" (Public Health 2021); "Epigen- 
etic variations due to nutritional status in early-life and its later impact on aging and disease" (Clin. Genet. 2020); "The Influence of Lifestyle Behaviors on the Incidence of Frailty" (J Frailty Aging, 2020); y "A Longitudinal Study on Multidimensional Resilience to Physical and Psychosocial Stress in Elderly Mexicans" (J Aging Health, 2020).

Dirección electrónica: rasamper@utmb.edu

Registro ORCID: https://orcid.org/0000-0002-2250-9401

\section{Rebeca Wong}

Profesora Investigadora en el Sealy Center on Aging y el Departamento de Medicina Preventiva; Directora del Centro Colaborador OMS/OPS para Envejecimiento y Salud, en la University of Texas Medical Branch. Es doctora en economía por la Universidad de Michigan y actuario de la Universidad Nacional Autónoma de México. Entre sus publicaciones recientes (con co-autores) destacan: "Health Insurance and the Aging: Evidence from the Seguro Popular program in Mexico (Demography 2018), "Dynamics of Economic Security among the Aging in Mexico: 2001-2012" (Population Research and Policy Review 2018), "Expansion of disability across successive Mexican birth cohorts: a longitudinal modelling analysis of birth cohorts born 10 years apart" ( $\mathrm{J}$ of Epidemiology \& Community Health 2019), "The effect of diabetes on the cognitive trajectory of older adults in Mexico and the U.S." (J of Gerontology: Psychol Sci Social Sci. 2020).

Dirección electrónica: rewong@utmb.edu

Registro ORCID: https://orcid.org/0000-0001-7287-0660

Artículo recibido el 4 de noviembre de 2020 y aprobado el 25 de enero de 2021. 